\title{
A novel electro-optical probe to diagnose plasma uniformity
}

\author{
M. Sarfaty, ${ }^{\text {a) }}$ M. Harper, and N. Hershkowitz \\ University of Wisconsin-Madison, Madison, Wisconsin 53706
}

(Received 8 January 1998; accepted for publication 25 June 1998)

\begin{abstract}
A novel electro-optical probe (EOP) has been developed to characterize the spatial uniformity of various plasma parameters. Spatially resolved electron density and energy distribution function, neutral and charged particle densities, as well as ion flow velocity are determined by the EOP. The design of the EOP combines a Mach probe, back-to-back charge collectors, and a collimated optical fiber. The light collection angle of the optical fiber is limited by recessing the fiber in a ceramic tube. The line-of-sight integration length of the plasma emission is bounded by the charge collector disk. A spatial resolution of $2.4 \mathrm{~cm}$ is obtained by the present design of the EOP. The ion flow velocity perpendicular to the charge collector surface is determined by the ratio of the ion saturation currents of the two counter facing charge collectors. Localized actinometry, that combines spatially resolved optical emission spectra and electron energy distribution functions, is used to determine the density of atomic chlorine and fluorine radicals. The spatial distribution is obtained by scanning the EOP across the plasma volume. (C) 1998 American Institute of Physics. [S0034-6748(98)03609-0]
\end{abstract}

\section{INTRODUCTION}

A major concern in plasma processing of materials is the spatial uniformity of the process. Process uniformity depends strongly on the spatial distribution of both charged and neutral particle densities and velocities as well as potentials. Process uniformity is a key requirement to enable large wafers or flat panels to be processed by plasma. The newly designed electro-optical probe (EOP) is developed to provide spatial information about the plasma which is would be more difficult to obtain by other methods. The EOP combines electrical and optical measurements of a spatially resolved plasma volume to resolve number density profiles of the plasma radicals. The simplicity of operation, the single small port access, and the relatively rapid derivation of information makes it a favorable technique.

Electrical probes ${ }^{1,2}$ have long been used in plasmas to measure electron density, electron energy distribution function (EEDF), potentials, and electromagnetic fields. In the present design of the EOP the plasma density, the EEDF, and the plasma and floating potentials are determined by backto-back disk charge collectors known as a Mach probe. ${ }^{3}$ This design provides information both outside and inside the spatially resolved plasma volume. The agreement between the measured plasma parameters in the two regions verifies that the EOP is causing a minimal perturbation to measurements in the spatially resolved volume. Furthermore, the back-toback disk collectors are used to determine the ion flow velocity perpendicular to the probe surface. When the two collectors are biased to draw ion saturation current, the difference in their collected current is interpreted as ion flow. The ion velocity is calculated from the ratio of the ion saturation currents $\mathrm{s}^{3,4}$ measured by the two counter facing disk collectors.

Optical emission spectroscopy (OES) has been exten-

${ }^{a)}$ Present address: Applied Materials, Santa Clara, CA. sively used in plasmas to obtain optical emission information which is integrated along the line-of-sight. To obtain spatial resolution, Abel inversion of a multidirectional OES is most frequently used assuming cylindrical symmetry of the plasma. The EOP is designed to collect localized light emission and charge particles. The optical emission is collected by an optical fiber that is recessed inside a ceramic tube. The localization of the OES is achieved by limiting the line-ofsight light collection volume by the planar charge-collector disk that faces the optical fiber. The acceptance angle of the optical fiber is limited and controlled by recessing the optical fiber inside a ceramic tube. The collected light emission is measured by an optical-multichannel analyzer placed at the output slit of a spectrometer. In this paper only line emission intensities are measured to obtain density profiles of neutral radicals.

Since the collected line emission is induced by the plasma electrons, knowledge of the local EEDF, measured by the probe, is essential for a correct interpretation of the OES signals as particle concentrations. The excitation rate ratio between the reference trace gas (argon/xenon) and the radical species (fluorine/chlorine), depends strongly on the details of the local EEDF. The dependence of the electron excitation rate ratios on the electron energy is an important part of the actinometry method ${ }^{5-9}$ used here to determine radical density.

\section{CHAMBER AND ELECTRO-OPTICAL PROBE SETUPS}

The experiments reported here are carried out in a cylindrical magnetically confined inductively coupled plasma (MCICP) tool. ${ }^{10}$ The plasma is produced by inductively coupled rf fields that are generated by a planar four turn antenna. The antenna is placed on top of a quartz window and centered around the chamber axis. The antenna and chamber diameters are 28 and $41 \mathrm{~cm}$, respectively, and the 


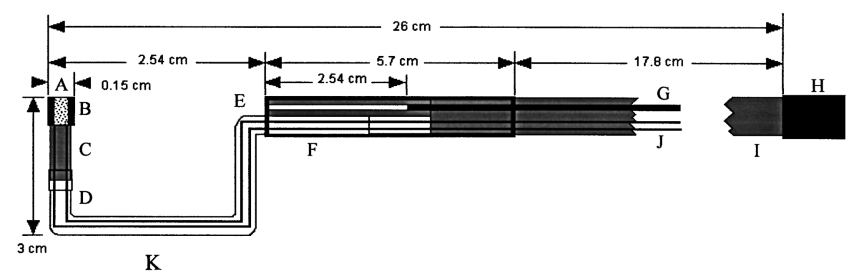

FIG. 1. Schematic design and dimensions of the electro-optical probe. A: Ceramic casting; B: back-to-back $3.18 \mathrm{~mm}$ o.d. tantalum disks $0.13 \mathrm{~mm}$ thick; C: $2.0 \mathrm{~mm}$ o.d. ceramic tube; D: Pyrex coupling tube; E: Single-bore ceramic collimator tube $1.5 \mathrm{~mm}$ i.d.; F: Pyrex tube $7.0 \mathrm{~mm}$ o.d.; G: Quartz fiber optic $1 \mathrm{~mm}$ o.d.; H: Stainless steel tubing $6.5 \mathrm{~mm}$ o.d.; I: Four-bore ceramic tubing $5.0 \mathrm{~mm}$ o.d.; J: Teflon coated wires; and K: Pyrex tubing 3.0 mm o.d.

chamber is $56 \mathrm{~cm}$ long. The magnetic enclosure of the chamber wall is formed by 24 azimuthally alternating magnetic poles equally distributed around the chamber. The $28 \mathrm{~cm}$ long pole assembly consists of ten individual magnets with magnetic field strength at the inner side of the chamber walls of $1 \mathrm{kG}$. The magnetic enclosure of the plasma enhances its density and improves its uniformity. A water cooled, unipolar electrostatic chuck with backside helium cooling is used to clamp a $100 \mathrm{~mm}$ diameter silicon wafer that is present during the experiments. One rf power supply and matching network is connected to the antenna $(13.56 \mathrm{MHz}$, up to 2.0 $\mathrm{kW})$ and a second to the wafer stage $(13.56 \mathrm{MHz}$, up to 0.5 $\mathrm{kW})$. The radial uniformity of high density low pressure $\left(10^{11} \mathrm{~cm}^{-3}, 4\right.$ mTorr) $\mathrm{CF}_{4}$ and $\mathrm{Cl}_{2}$ plasmas are studied.

The EOP, shown in Fig. 1, consists of two back-to-back tantalum disks, that serve as charge collectors to measure the local electrical properties of the plasma, and an optical fiber that collects light emission from a spatially resolved volume. Three bores out of the four-bore ceramic tube carry two wires, which are connected to the planar disks, and a $1 \mathrm{~mm}$ diameter quartz fiber. The planar disks are $3.2 \mathrm{~mm}$ in diameter $0.13 \mathrm{~mm}$ thick and are separated by $1.5 \mathrm{~mm}$ of ceramic casting material. The inner disk is placed $24 \mathrm{~mm}$ in front of a single-bore ceramic tube that extends out of the bore that carries the optical fiber. The single-bore tube is used to limit the fiber acceptance angle to less than $1.5^{\circ}$. By back-lighting the optical fiber and recessing it inside the ceramic tube we limit the spot size illuminated by the fiber to be smaller than the diameter of the inner disk collector that faces the fiber. Therefore, the optical emission is collected from a column $24 \mathrm{~mm}$ long and $2 \mathrm{~mm}$ wide. The light collection volume can be adjusted by moving the fiber inside the collimating ceramic tube. The coupling of scattered light as well as reflected light from the tantalum disk to the optical fiber is negligible relative to the light collection from the spatially resolved volume.

The U-shape end of the probe is made of Pyrex glass tube $3 \mathrm{~mm}$ diameter enclosing the Teflon coated wires that are connected to the planar disks. The U-shape geometry is needed to reduce the perturbations of a nearby surface on the properties of the spatially resolved plasma. A grounded stainless steel tube covers the four-bore ceramic tube to reduce rf noise of the electrical signals. In the MCICP, the antenna induced rf noise near the wafer site, $27 \mathrm{~cm}$ from the antenna, is relatively small and is further reduced from the

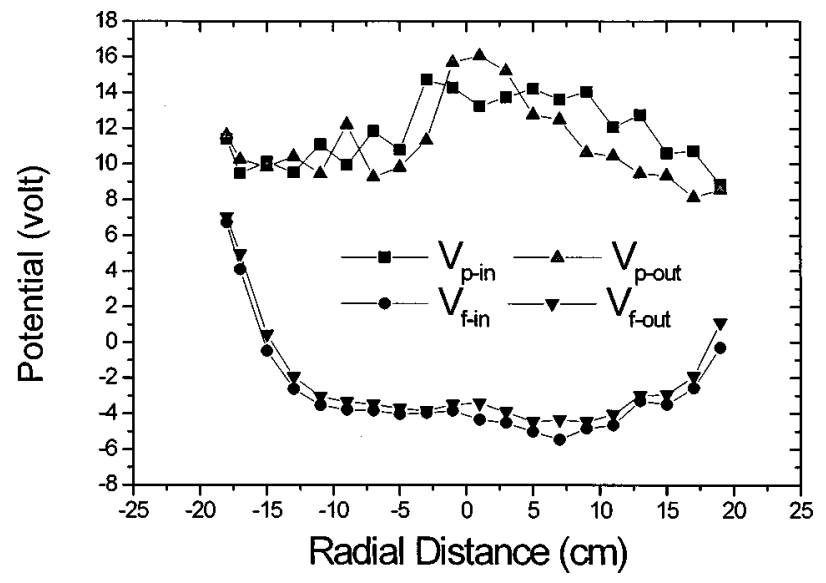

FIG. 2. Radial plasma and floating potential distribution. Inner and outer probe are the charge collecting disks facing the optical fiber or the volume restricted plasma and the plasma outside this region, respectively.

probe current-voltage $(I-V)$ traces by a low-pass and band rejecting filters. In this article the probe is inserted into the plasma through a side port and scanned across the chamber diameter to obtain radial plasma uniformity.

The OES signals are acquired by a $30 \mathrm{~cm}$ spectrometer with a grating of 1200 grooves $/ \mathrm{mm}$. A water-cooled nitrogen-purged optical multichannel analyzer of an intensified charge-coupled device (CCD) array records the light signals at the output slit of the spectrometer. A spectral resolution of $\sim 0.06 \mathrm{~nm} / \mathrm{pixel}$ is obtained in the spectral range of $700-850 \mathrm{~nm}$. Gas mass spectrometry data is obtained by a residual gas analyzer (RGA). The RGA was calibrated before and after the experiments by flowing argon and xenon gases according to the experiment runs. The calibration provides a relation between the RGA and the ionization-gauge pressure readouts, i.e., absolute number density or pressure for the RGA readouts for the nobel gases. The density of chlorine or fluorine radicals are determined by introducing into the discharge $4 \%$ of xenon or argon gas, respectively. The measurements described in this article are made at an axial distance of $\sim 1 \mathrm{~cm}$ above a silicon wafer. The radial scans are taken in random order across the entire chamber. The spatially resolved radical densities are compared with line-of-sight integrated OES collected from a cylindrical plasma volume of $\sim 1 \mathrm{~cm}$ diameter centered $\sim 1 \mathrm{~cm}$ above the wafer. The line integrated measurements are done through a side window using a lens to collimate the fiber light collection.

\section{CHARACTERIZATION OF PLASMA UNIFORMITY}

The spatial distribution of the plasma $\left(V_{p}\right)$ and floating $\left(V_{f}\right)$ potentials are determined by both the inner and outer disk collectors of the EOP. The radial potential variations in chlorine plasma, shown in Fig. 2, is measured at a distance of $1 \mathrm{~cm}$ above the wafer. The floating potential is obtained from the zero crossing of the $I-V$ trace, while the plasma potential is determined from the zero crossing of its second derivative. The plasma potential decreases towards the walls while the floating potential increases. As seen in Fig. 2, the 


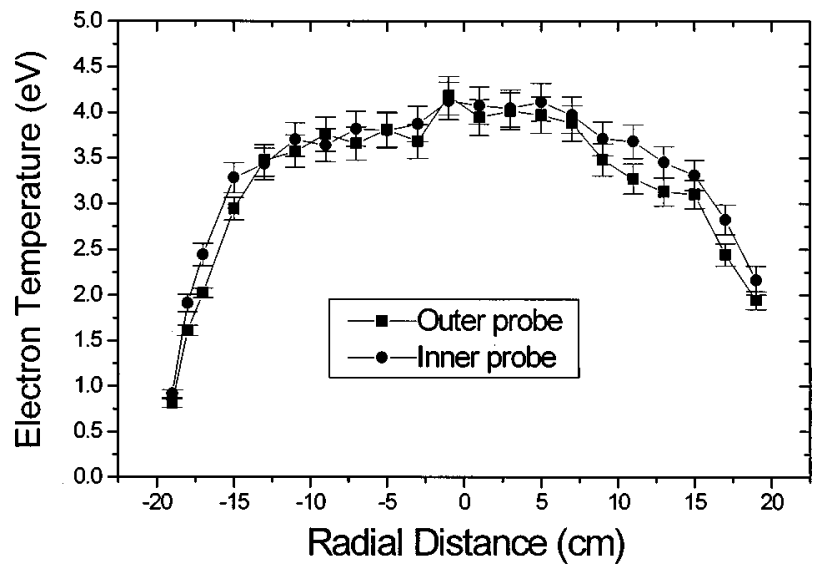

FIG. 3. Radial electron temperature profile determined by both inner and outer probes measured inside and outside the plasma restricted volume.

similar potentials obtained by both charge collectors indicate similar plasma conditions in the open and resolved plasma volume.

The electron temperature was evaluated both by fitting a Maxwellian energy distribution function to the second derivative of the $I-V$ curve and by fitting an exponential function to the electron $I-V$ trace in the voltage range below the plasma potential, i.e., the electron retarding part. The radial dependence of the electron temperature, shown in Fig. 3, is obtained by the former method. A higher electron temperature is measured at the center of the chamber with a small decreasing gradient towards $15 \mathrm{~cm}$ from the center. A steeper gradient is observed beyond $15 \mathrm{~cm}$ radius, at a distance of 5 $\mathrm{cm}$ from the chamber wall. This distance is close to the outer edge of the rf antenna and to where the magnetic enclosure field corresponds to the ion gyroradius. An almost constant relation is observed between the sheath potential $V_{s}=V_{p}$ $-V_{f}$ and the electron temperature of $V_{s} / T_{e} \sim 4.5$ across the chamber diameter. The inner and outer charge collector traces yield similar electron temperature profile within the experimental error. A slightly higher temperature is obtained by the inner collector at radial distances larger than $15 \mathrm{~cm}$.

The radial profile of the electron density, shown in Fig. 4 , is obtained from the integration of the second derivative of the $I-V$ curve. The radial profile of the plasma density

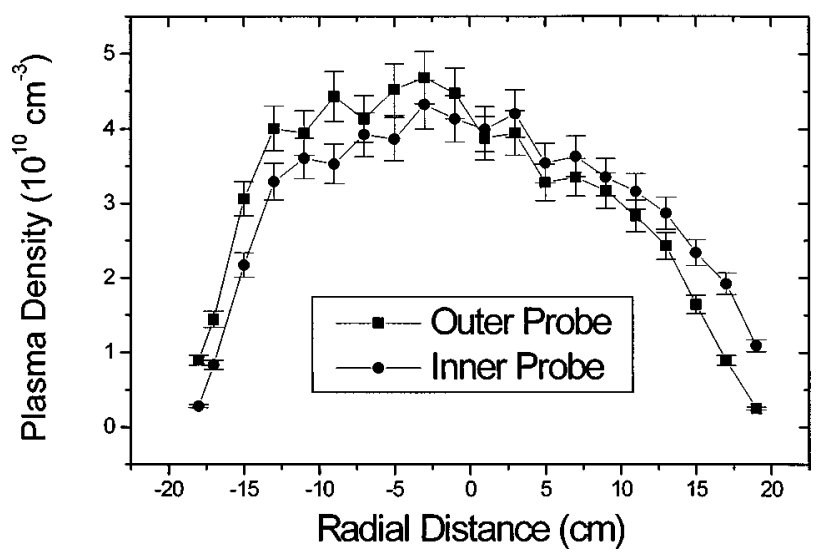

FIG. 4. Radial electron density profile determined by the inner and the outer probes measured inside and outside the plasma restricted volume.
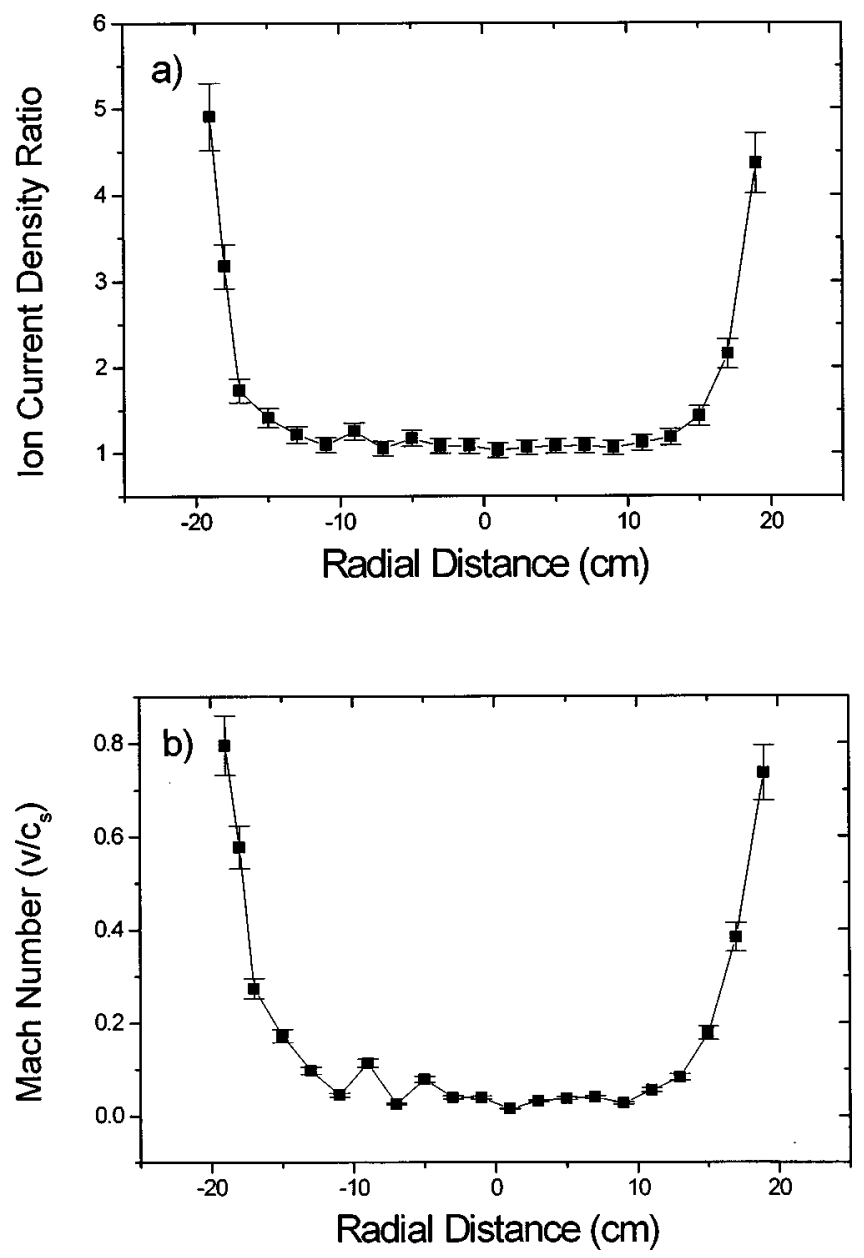

FIG. 5. (a) Radial profile of the ion flux ratio in the radial direction. (b) Radial ion velocities measured in Mach numbers as a function of radial position.

shows small density gradients in the center of the chamber and an increasing gradient close to the chamber walls. It is important to note that the radial density profiles obtained by the two charge collectors cross each other around the chamber center. The density determined by the collector that faces the nearest chamber wall is typically lower than the opposite collector. A similar behavior is also observed in the ion saturation current measured by the two charge collectors. This difference is attributed to the radial component of the ion flow. A lower density is measured by the collector that is in the shadow of the radial flow.

The ion current density ratio of the two collectors can be used to determine the component of the ion flow velocity perpendicular to the disk surface which is radial in this case. Similarly, if the probe is introduced along the axial direction, the axial component can be determined. The radial ion current density ratio, shown in Fig. 5(a), indicates a larger radial ion flow close to the chamber walls. The ion Mach number, $M=V_{i} / C_{s}$, or ion velocity, $V_{i}$, normalized to the ion sound speed, $C_{s}=\left(T_{e} / M_{i}\right)^{1 / 2}$, shown in Fig. 5(b), is calculated from the ion current density ratios assuming a pure singly ionized $\mathrm{Cl}$ plasma. The relation between the ion current density ratio, $R$, and the ion Mach number is given by $R$ $=\exp (2 M)$ derived from the Mach probe theory. ${ }^{3,4}$ The radial profile of the measured electron temperature, shown in 
Fig. 3, is required to transfer the ion Mach number to radial ion velocities. In spite of the approximately fourfold drop in the electron temperature near the walls the radial ion velocities increase as the plasma approaches the chamber walls. The measured radial component of the ion velocities reflect the radial gradients of the plasma density profile, shown in Fig. 4, and indicative of the plasma presheath near the chamber walls. To better evaluate the ion radial velocity, a Doppler shift OES or laser induced fluorescence of the main plasma ion should be measured and compared with the Mach relation used above.

The optical part of the EOP collects line emission induced by the plasma from a resolved volume, restricted by the disk collector and the optical fiber acceptance angle which is collimated by the ceramic tube it is recessed in. A technique known as actinometry ${ }^{5-9}$ is used to determine the concentration of reactive species such as fluorine and chlorine in this experiment. Actinometry uses the emission of a nonreactive noble gas, added in trace amounts to the discharge, to determine the density of a relevant species from its emission intensity. The underlying assumption is that the emission of both the actinometer and the target particle are produced by electron impact excitation from the ground state species. Therefore, the electron-impact cross sections of both species need to have similar threshold energy and shape as a function of the electron energy. Also, the excited states of both species must decay primarily by photon emission. The inert actinometer gases used here are argon and xenon for fluorine and chlorine density measurements, respectively. To obtain absolute number densities, the amount of the trace gas was measured with a pressure calibrated RGA set at one of the chamber ports. The radial uniformity of the actinometer was confirmed by measuring the local density of the actinometer with a $100 \mathrm{~cm}$ long quartz tube of $0.9 \mathrm{~cm}$ diameter that was scanned across the chamber diameter. A uniform distribution was observed across the chamber for an operating pressure of 4 mTorr both prior and during the plasma discharge. The spatial distribution of the actinometer is important when the local density of the target radical needs to be determined.

The emission intensity of species $x, I_{x}$, is given by

$$
I_{x}=C_{d}(\lambda) n_{g x} n_{e} K_{x} Q_{x} b_{\lambda x},
$$

where $C_{d}(\lambda)$ is the detector spectral response, $n_{g x}$ is the ground state density of species $x, n_{e}$ the electron density, $K_{x}$ is the electron excitation rate from the ground state to the observed level, $Q_{x}$ is the quantum yield for photon emission, which is unity at process pressure of $4 \mathrm{mTorr}$, and $b_{\lambda x}$ is the emission branching ratio for the transitions used. Relating the emission intensities of the target radical to that of the actinometer yields the following expression for the radical ground state population:

$$
n_{g x}=n_{\text {act }} \frac{I_{x}}{I_{\text {act }}} \times \frac{K_{\text {act }}\left(T_{e}\right)}{K_{x}\left(T_{e}\right)} \times \frac{C_{\text {act }} b_{\lambda \text { act }}}{C_{x} b_{\lambda x}} .
$$

The ratio of branching ratios in the last term is available from atomic transition tables and the wavelength response of the detector within the spectral range of $700-830 \mathrm{~nm}$ is relatively constant. The number density of the actinometer was

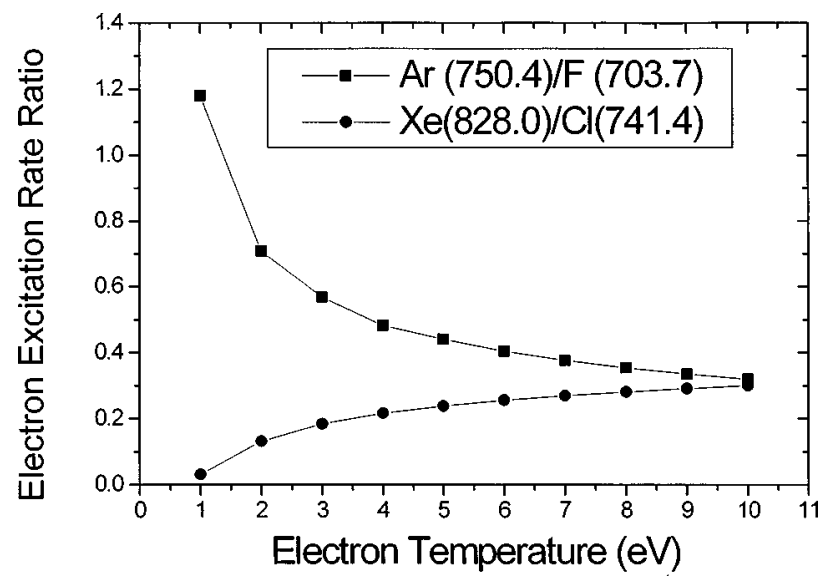

FIG. 6. Ground state electron impact excitation rate ratios as a function of the electron temperature. All the transitions are from the ground state to the observed light emitting level fluorine $\left(3 p[3 / 2]^{2} P\right)$ and $\operatorname{argon}\left(2 P_{1}\right)$ and chlorine $\left(4 p[3 / 2]^{4} P\right)$ and xenon $\left(2 P_{5}\right)$.

measured with an online calibrated RGA stable to within $\pm 1 \%$ during data acquisition. The line intensities were measured at various radial positions selected in random order across the chamber diameter. The measurement of the line emission intensity is averaged over 20 data acquisition samples captured at $100 \mathrm{~ms}$ exposure time of the intensified CCD array. Both the spatially resolved and line-of-sight integrated emission intensity show repeatability of $\pm 3 \%$ between successive data acquisitions.

The electron impact excitation cross sections, $\sigma_{x}$, from the ground state to the energy level observed are taken from experimentally available sources for xenon ${ }^{11}$ and $\operatorname{argon}^{12}$ and computed for argon, ${ }^{13}$ fluorine, ${ }^{13}$ and chlorine. ${ }^{13}$ The excitation rate for a Maxwellian EEDF from the ground state to the observed level, $E_{\text {thx }}$, are calculated using Eq. (3):

$$
K_{x}=\frac{1}{(m \pi)^{1 / 2}}\left(\frac{2}{T_{e}}\right)^{3 / 2} \int_{E_{\mathrm{thx}}}^{\infty} \sigma_{x}(\epsilon) \epsilon e^{-\epsilon / T_{e}} d \epsilon
$$

The ratios of excitation rates as a function of electron temperature are shown in Fig. 6 both for argon $\left(1 S_{2}-2 P_{1}\right.$ $750.4 \mathrm{~nm})$ to fluorine $\left(3 s[3 / 2]^{2} P-3 p[3 / 2]^{2} P 703.7 \mathrm{~nm}\right)$ and xenon $\left(1 S_{4}-2 P_{5} 828.0 \mathrm{~nm}\right)$ to chlorine $\left(4 s[5 / 2]^{2} P\right.$ $\left.-4 p[3 / 2]^{4} P 741.4 \mathrm{~nm}\right)$. The spectral lines selected are those whose upper level populations are the least affected by metastable levels. ${ }^{14}$ The temperature dependence of these ratios is very important when the number density of the relevant radical needs to be determined using Eq. (2). The radial profile of the electron temperature, determined by fitting a Maxwellian to the measured EEDF, is used here to calculate the radial dependence of the excitation rate ratios. It is important to note that for a case of a non-Maxwellian EEDF the measured EEDF can be used in Eq. (3) to calculate the excitation rates.

The radially resolved and the line integrated emission intensity ratios of fluorine to argon are given in Fig. 7(a) with an upper limit error bar of $\pm 6 \%$ resulting from line intensity repeatability. The radial profile of the partial pressure of atomic fluorine in the discharge, determined by Eq. (2), is shown in Fig. 7(b). The $\pm 10 \%- \pm 17 \%$ range of the 

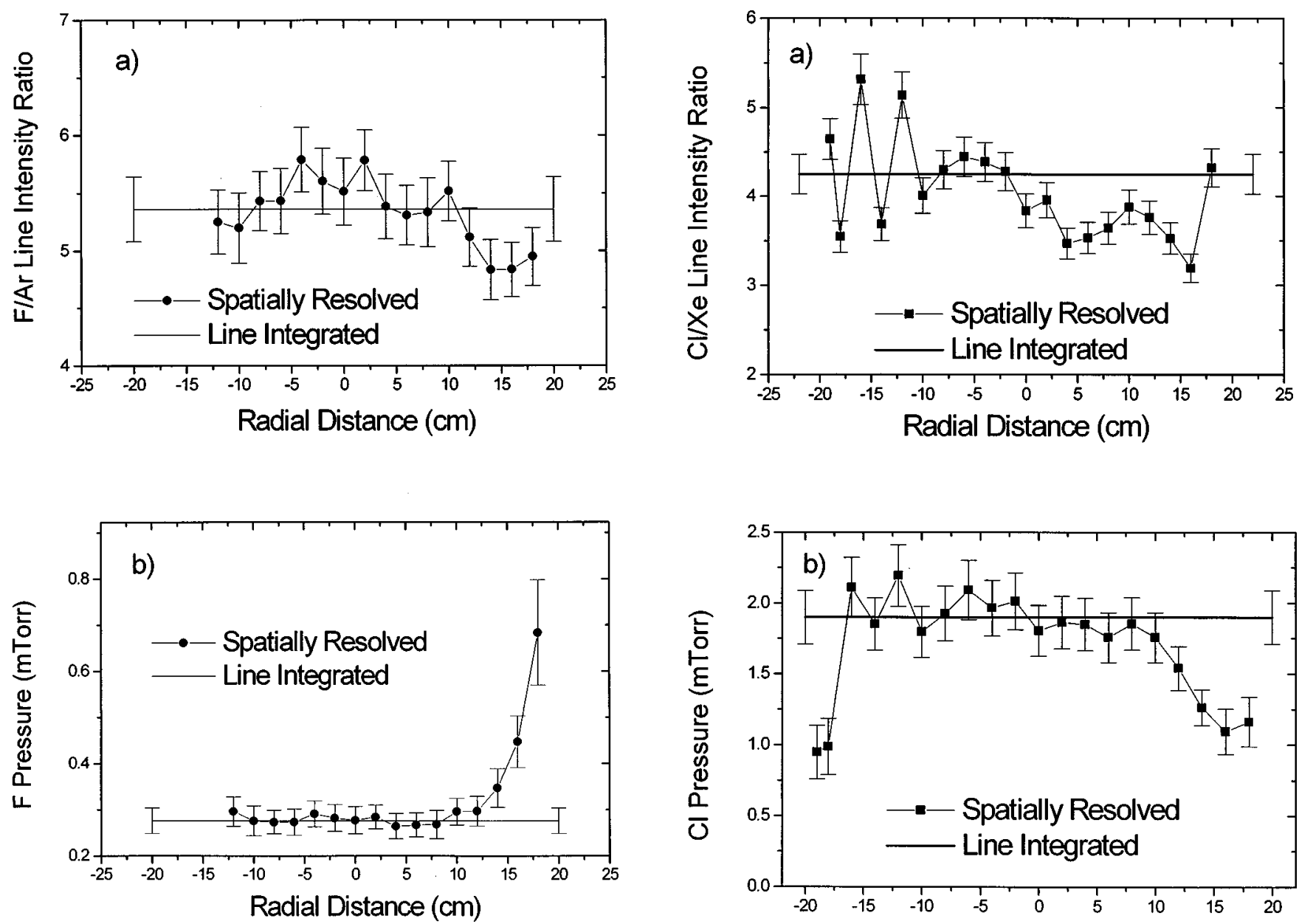

FIG. 7. (a) Radial profile of the emission intensity ratio of fluorine (703.7 $\mathrm{nm})$ to argon $(750.4 \mathrm{~nm})$ spectral lines. (b) Radial distribution of the partial pressure of fluorine.

error bars of the fluorine radial pressure profile results from the uncertainty in both the line intensity ratios and the electron temperature. A clear rise in atomic fluorine partial pressure is observed near the chamber walls. A possible explanation is that ions impinging on the chamber walls which are coated with fluorine containing films can introduce atomic fluorine into the plasma. The line integrated emission intensity ratio measured through a side window agrees with the radially resolved ratios in the bulk of the plasma. The absolute partial pressure of atomic fluorine is determined from the argon partial pressure measured during the scans with the calibrated RGA.

The line intensity ratios of chlorine to xenon as a function of radial position are shown in Fig. 8(a) with and upper limit error bars of $\pm 6 \%$. The radial profile of atomic chlorine partial pressure, shown in Fig. 8(b), is obtained by Eq. (2), accounting for the radial variations of the excitation rate ratios as a function of the measured electron temperature profile. The partial pressure of atomic chlorine is lower near the chamber walls than in the bulk of the plasma, due to wall recombination of atomic chlorine into molecular chlorine. The ratio of the line integrated emission intensity agrees with the radially resolved ratios measured in the bulk of the plasma.

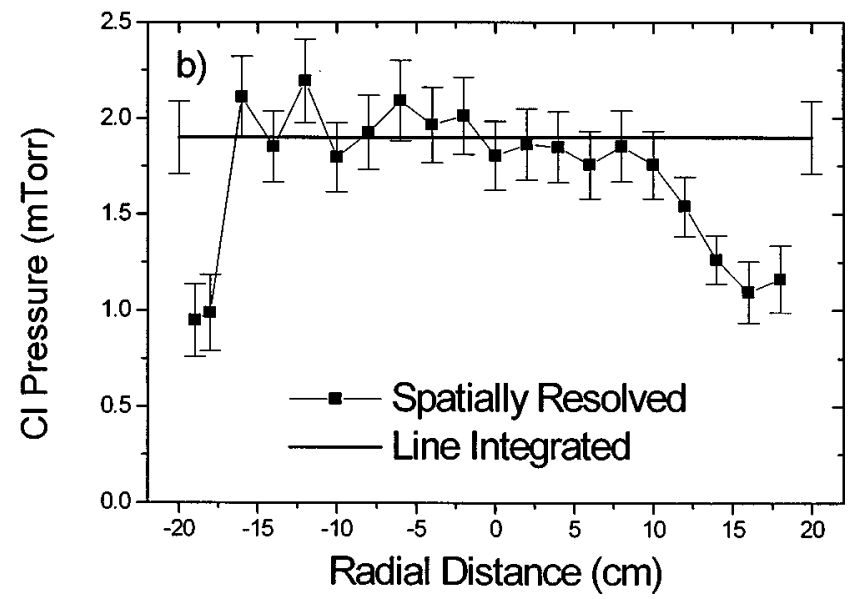

FIG. 8. (a) Radial profile of the emission intensity ratio of chlorine (741.4 $\mathrm{nm})$ to xenon $(828.0 \mathrm{~nm})$ spectral lines. (b) Radial distribution of the partial pressure of chlorine.

\section{ACKNOWLEDGMENTS}

The authors would like to thank K. Kriewaldt for his technical assistance in the construction of the electro-optical probe. This work was supported by the National Science Foundation under Grant No. EEC-8721545.

${ }^{1}$ F. F. Chen, in Plasma Diagnostic Techniques, edited by R. H. Huddlestone and S. L. Leonard (Academic, New York, 1965), pp. 113-200.

${ }^{2}$ L. Schott, in Plasma Diagnostics, edited by W. Lochte-Holtgreven (North-Holland, Amsterdam, 1968), pp. 668-731.

${ }^{3}$ I. H. Hutchinson, Principles of Plasma Diagnostics (Cambridge University Press, New York, 1987).

${ }^{4}$ K.-S. Chung and I. H. Hutchinson, Phys. Rev. A 38, 4721 (1988).

${ }^{5}$ J. W. Coburn and M. Chen, J. Appl. Phys. 51, 3134 (1980).

${ }^{6}$ R. d'Agostino, F. Cramarossa, S. DeBenedictus, and G. Ferraro, J. Appl. Phys. 52, 1259 (1981).

${ }^{7}$ V. M. Donnelly, D. L. Flamm, W. C. Dautremont-Smith, and D. Werder, J. Appl. Phys. 55, 242 (1984)

${ }^{8}$ R. A. Gottscho and V. M. Donnelly, J. Appl. Phys. 56, 245 (1984).

${ }^{9}$ A. D. Richards, B. E. Thompson, K. D. Allen, and H. H. Sawin, J. Appl. Phys. 62, 792 (1987).

${ }^{10}$ C. Lai, B. Brunmeier, and R. C. Woods, J. Vac. Sci. Technol. A 13, 2086 (1995).

${ }^{11}$ P. V. Fel'tsan and I. P. Zapesochnyi, Ukr. Phys. J. 13, 143 (1968).

${ }^{12}$ I. P. Zapesochnyi and P. V. Fel'tsan, Opt. Spectra 20, 521 (1966).

${ }^{13} \mathrm{Y}$. Ralchenko (private communication).

${ }^{14}$ M. V. Malyshev and V. M. Donnelly, J. Vac. Sci. Technol. A 15, 550 (1997). 